\title{
Underestimated Effect of a Polymer Matrix on the Light Emission of Single $\mathrm{CsPbBr}_{3}$ Nanocrystals
}

Gabriele Rainò, ${ }^{\dagger, \ddagger}$ Annelies Landuyt, ${ }^{\dagger, \ddagger}$ Franziska Krieg, ${ }^{\dagger, \dagger}$ Caterina Bernasconi, ${ }^{\dagger, \dagger}$

Stefan T. Ochsenbein, ${ }^{\dagger, \ddagger \odot}$ Dmitry N. Dirin, ${ }^{\dagger, \ddagger \odot ~ M a r y n a ~ I . ~ B o d n a r c h u k, ~}$ and Maksym V. Kovalenko*,

${ }^{\dagger}$ Institute of Inorganic Chemistry, Department of Chemistry and Applied Biosciences, ETH Zürich, CH-8093 Zürich, Switzerland ${ }^{\ddagger}$ Laboratory for Thin Films and Photovoltaics, Empa - Swiss Federal Laboratories for Materials Science and Technology, CH-8600 Dübendorf, Switzerland

\section{Supporting Information}

ABSTRACT: Lead-halide perovskite $\mathrm{APbX}_{3}(\mathrm{~A}=\mathrm{Cs}$ or organic cation; $\mathrm{X}=\mathrm{Cl}, \mathrm{Br}, \mathrm{I}$ ) nanocrystals (NCs) are the subject of intense research due to their exceptional characteristics as both classical and quantum light sources. Many challenges often faced with this material class concern the longterm optical stability, a serious intrinsic issue connected with the labile and polar crystal structure of $\mathrm{APbX}_{3}$ compounds. When conducting spectroscopy at a single particle level, due to the highly enhanced contaminants (e.g., water molecules, oxygen) over the $\mathrm{NC}$ ratio, deterioration of $\mathrm{NC}$ optical

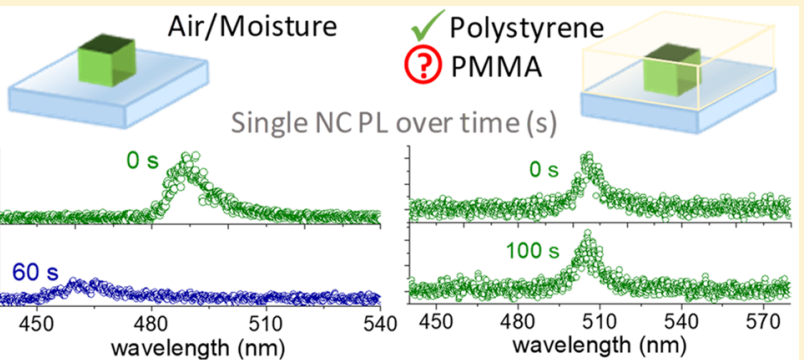
properties occurs within tens of seconds with typically used excitation power densities $\left(1-100 \mathrm{~W} / \mathrm{cm}^{2}\right)$ and in ambient conditions. Here, we demonstrate that choosing a suitable polymer matrix is of paramount importance for obtaining stable spectra from a single NC and for suppressing the dynamic photoluminescence blueshift. In particular, polystyrene (PS), the most hydrophobic among four tested polymers, leads to the best optical stability, one to two orders of magnitude higher than that obtained with poly(methyl methacrylate), a common polymeric encapsulant containing polar ester groups. Molecular mechanics simulations based on a force-field approximation corroborate the hypothesis that PS affords for a denser molecular packing at the NC surface. These findings underscore the often-neglected role of the sample preparation methodologies for the assessment of the optical properties of perovskite NCs at a single-particle level and guide the further design of robust single photon sources.

KEYWORDS: Perovskite nanocrystals, single quantum dots, optical properties, microphotoluminescence, photon statistics

$\mathrm{N}$ anocrystals (NCs) of perovskite lead halides $\left[\mathrm{APbX}_{3}, \mathrm{~A}=\right.$ formamidinium (FA), methylammonium (MA) or Cs; $\mathrm{X}$ $=\mathrm{Cl}, \mathrm{Br}, \mathrm{I}]$ have recently been synthesized with precise control over their size and composition, offering bright, tunable, and narrowband photoluminescence (PL) over the entire visible spectral range. ${ }^{1-10}$ This new material system opened new opportunities to boost the performance of photonic devices based on quantum nanomaterials. ${ }^{11-13}$ Owing to their intrinsic defect tolerance, perovskite NCs had been employed in highly efficient LEDs ${ }^{14-18}$ and as lasing medium. ${ }^{11,19-21}$

Single NC optical spectroscopy probes the intrinsic optical properties and overcomes the limitations of ensemble measurements by eliminating the effects of NC size- and composition inhomogeneities. This technique had been recently used to study the optical properties of perovskite NCs at cryogenic and ambient conditions. At cryogenic temperatures, the emission of single $\mathrm{CsPbX}_{3} \mathrm{NCs}$ is nearly blinking-free ${ }^{22-24}$ and with ultrafast radiative decay. ${ }^{22}$ The emission originates from a bright triplet manifold, ${ }^{23,25-27}$ and the measurements with magnetic field have unveiled the charged excitons dynamics ${ }^{23}$ and the presence of the Rashba effect. ${ }^{24}$ While initial efforts concentrated mainly on $\mathrm{CsPbX}_{3}$ NCs, more recent reports concerned hybrid organic-inorganic perovskite $\mathrm{NCs}\left(\mathrm{FAPbX}_{3}, \mathrm{X}=\mathrm{Br}, \mathrm{I}\right)$, revealing, among other features, an enhanced exciton-optical phonon interaction. ${ }^{28,29}$

Operation at room temperature is a highly desired prerequisite for the development of quantum light sources (e.g., single-photon $\mathrm{LEDs}^{30}$ ) for quantum information technologies. ${ }^{31}$ At room temperature, $\mathrm{APbX}_{3} \mathrm{NCs}$ have been shown to emit a regular stream of single photons with very high single photon purity, as evidenced by the antibunching in the second-order correlation function $\left[g^{2}(0)<0.1\right] .^{32-38}$ These NCs are presently nearly unmatched in terms of spectral tunability and facile solution synthesis and processability. However, despite their great potential, single perovskite NCs still suffer from insufficient optical stability in ambient

Received: February 16, 2019

Revised: $\quad$ May 1, 2019

Published: May 22, 2019 
a

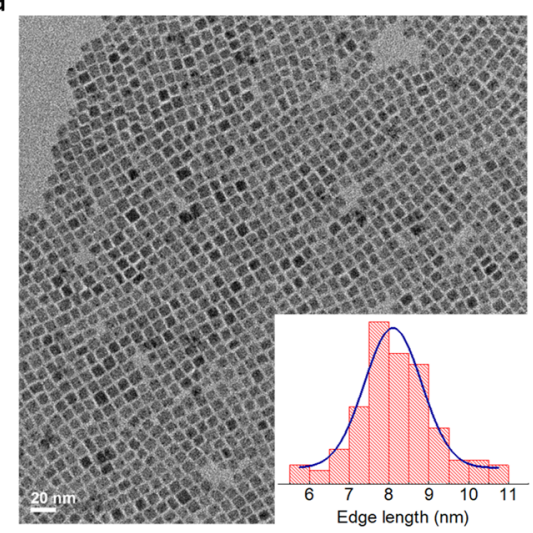

b

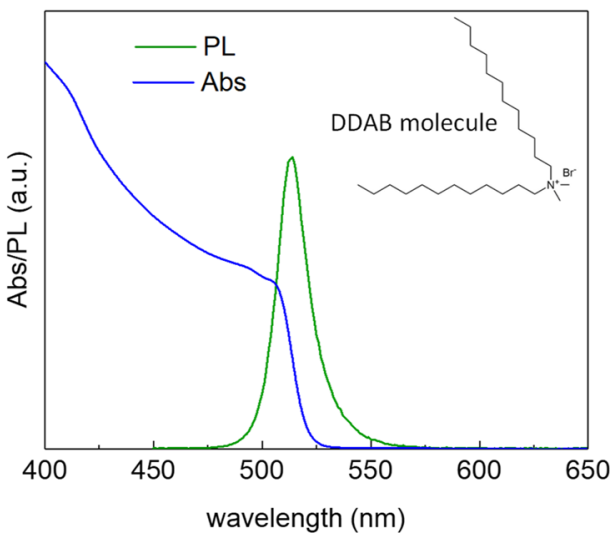

Figure 1. (a) Low-magnification transmission electron microscopy image of $\mathrm{CsPBBr}_{3}$ NCs. Statistical analysis of size distribution reveals a mean edge length of $8 \mathrm{~nm}$. (b) Typical RT absorption and emission spectra obtained in solution. The inset shows the molecular structure of a DDAB molecule used for the postsynthetic treatment of the NC solutions.
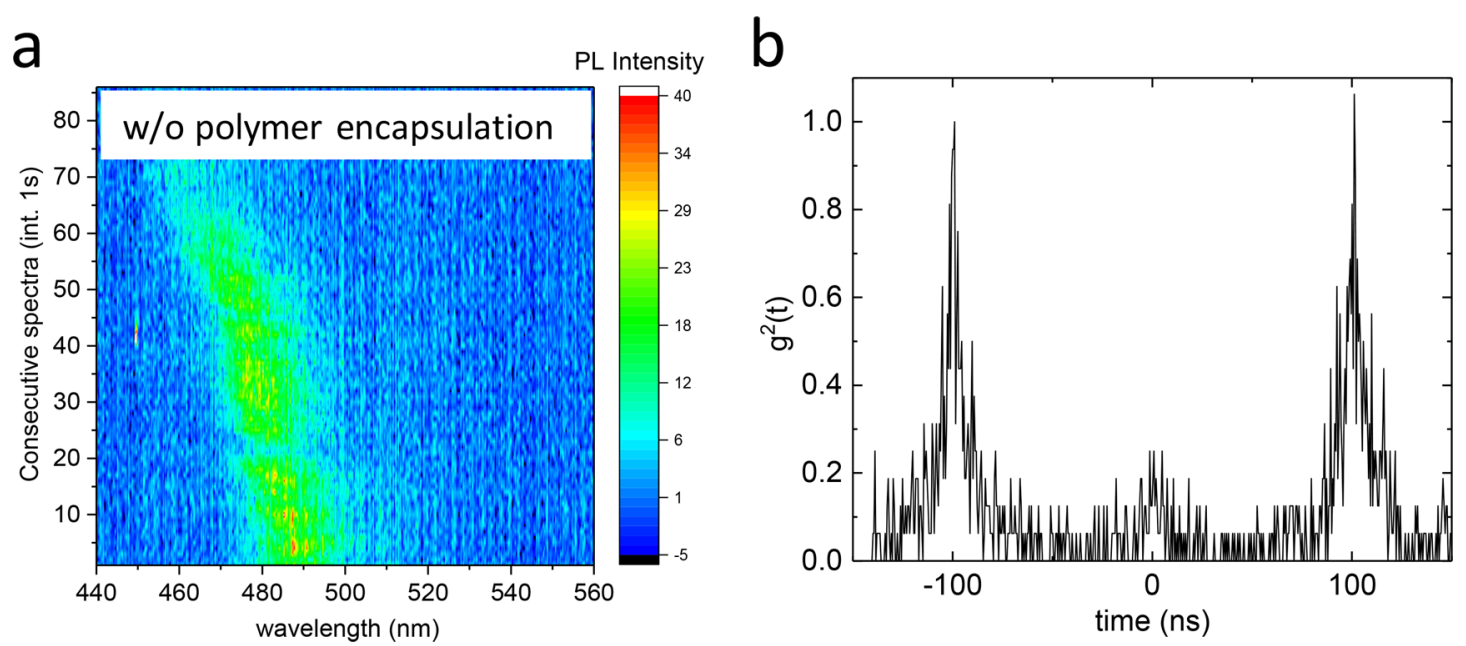

Figure 2. Experiments with "bare" CsPbBr ${ }_{3} \mathrm{NCs}$ (without polymer encapsulation). (a) The two-dimensional colored plot of several consecutive PL spectra obtained with an integration time of $1 \mathrm{~s}$. The $\mathrm{CsPbr}_{3} \mathrm{NCs}$ were spin-coated onto a glass substrate and were in direct contact with air. (b) Second-order correlation measurement $\left(\mathrm{g}^{2}(t)\right)$ displaying an antibunching behavior, characteristic of single photon emission.

conditions. $^{33,39-42}$ Moisture-assisted photodegradation is widely regarded as a major cause of instability, in addition to the thermal and solvent lability of these materials. ${ }^{43-46}$ At the single particle level, the dynamic blueshift of the PL emission and a concomitant PL quenching were indeed attributed to the photoaccelerated reaction with atmospheric water. ${ }^{39}$

In this Letter, we demonstrate an effective encapsulation procedure that enhances the NC optical stability at the single particle level. The dynamic PL blueshift is strongly suppressed by embedding single NCs in a polymeric matrix of polystyrene (PS) by up to several orders of magnitude as compared to the other studied polymers. High hydrophobicity and efficient molecular packing of PS with the long-chain NC surface ligands are found as key factors for protecting NCs. Somewhat counterintuitive, the overall water permeability of a polymer, a characteristic widely regarded as a measure for the utility of the polymer to act as a water barrier, did not correlate with the observed stabilities of NCs. In particular, TOPAS (a cyclic olefin copolymer) with a water permeability of $0.035 \mathrm{~g} \cdot \mathrm{mm} /$ $\left(\mathrm{m}^{2} \cdot \mathrm{d}\right)$, a factor of 50 lower than that of PS, did not lead to a notable improvement. Importantly, poly(methyl methacrylate) (PMMA), a commonplace matrix for single NC experiments, afforded only an order of magnitude lower NC stability; this can be attributed to a nonoptimal interfacial contact with the ligands, caused by the presence of polar ester groups, resulting in only partial coverage of the NC volume. These findings shed light on degradation processes in perovskite NCs and offered new strategies for the development of nonclassical lightemitting devices with strongly improved optical stability.

As a test-bed material, we have used $\mathrm{CsPbBr}_{3} \mathrm{NCs}$, synthesized following a similar procedure as previously reported. ${ }^{1}$ A postsynthetic treatment with didodecyldimethylammonium bromide (DDAB) molecules has been used to increase the stability and PL quantum yield $(\mathrm{QY})^{10}$ (see Supporting Information (SI)). DDAB-capping had been previously shown to result in a more static surface coverage suitable for obtaining purified colloids unlike that of the parent ligands used for the synthesis (oleic acid and oleylamine) ${ }^{10}$ As shown in Figure 1a, narrowly dispersed cubic NCs have a mean size of $8 \mathrm{~nm}$ with less than $15 \%$ standard deviation. Roomtemperature (RT) absorption and emission spectra are reported in Figure 1b, showing excitonic absorption profile and narrowband PL (full width at half-maximum of $17 \mathrm{~nm}$; for 

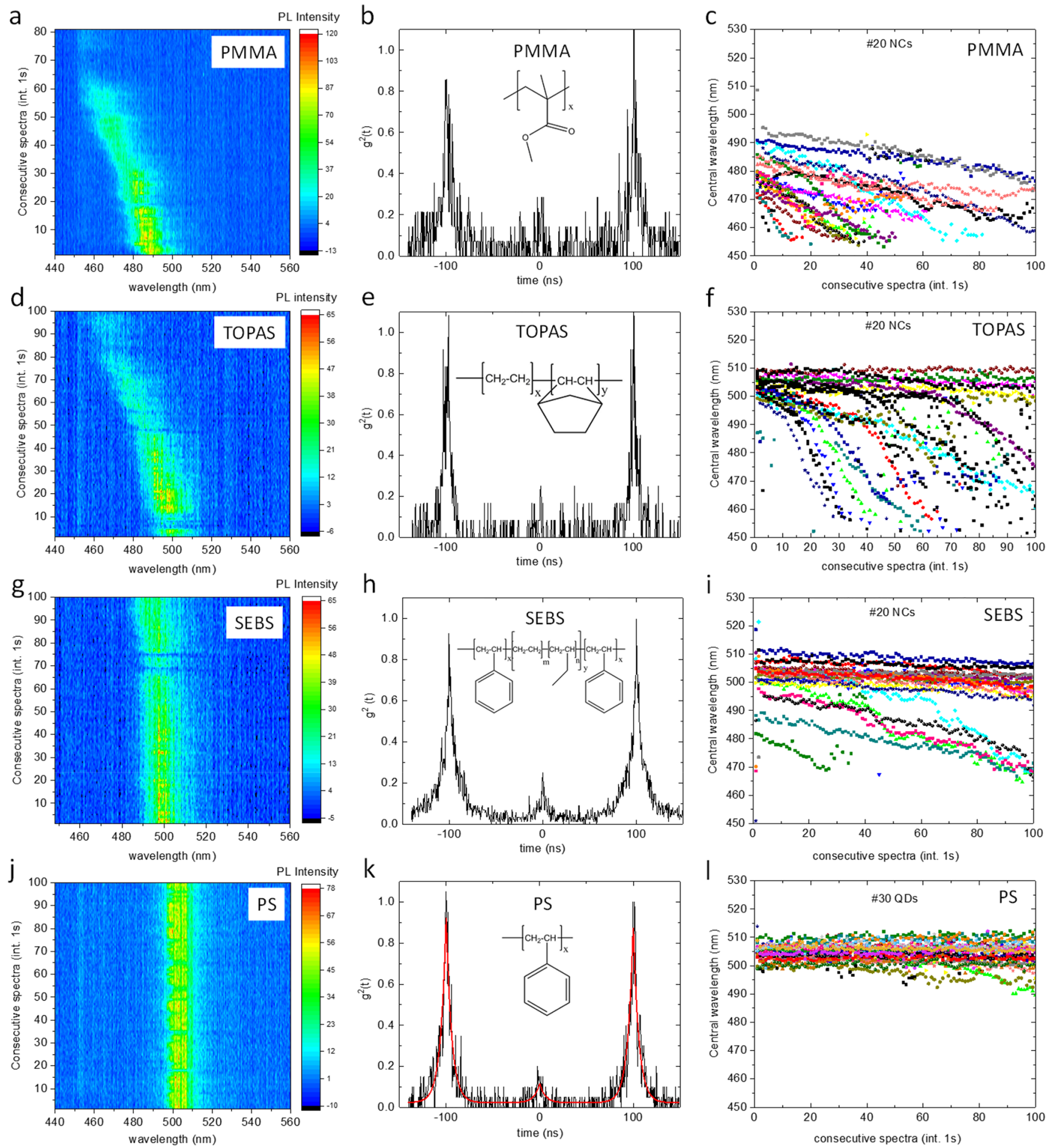

Figure 3. (a,d,g,j) The two-dimensional colored plots of several consecutive PL spectra obtained with NCs embedded in PMMA, TOPAS, SEBS, and PS, respectively. (b,e,h,k) Second-order correlation measurements obtained for a single NC embedded into PMMA, TOPAS, SEBS, and PS, respectively. The inset reports the chemical structure of the different polymers. Solid red line in (k) is the best fit obtained using the sum of three exponential decay functions. (c,f,i,l) Extracted central emission wavelength for different NCs embedded in PMMA, TOPAS, SEBS, and PS, respectively.

example, $77 \mathrm{meV}$ ). These NCs feature PL QY higher than $90 \%$ in toluene solution.

To study the optical stability, single NC spectroscopy was performed with a home-built microscope. The samples were excited by a $405 \mathrm{~nm}$ pulsed laser (repetition rate of $10 \mathrm{MHz}$; excitation power density of $\left.70 \mathrm{~W} / \mathrm{cm}^{2}\right)$, focused by an oil- immersion objective $(\mathrm{NA}=1.3)$. PL was detected by a $0.5 \mathrm{~m}$ monochromator coupled to an electron multiplying chargecoupled device camera. A diluted solution of NCs was mixed with a solution containing the polymer under examination and spin-coated on a glass coverslip (see SI for more details). As a reference sample, a diluted solution of NCs was directly spin- 


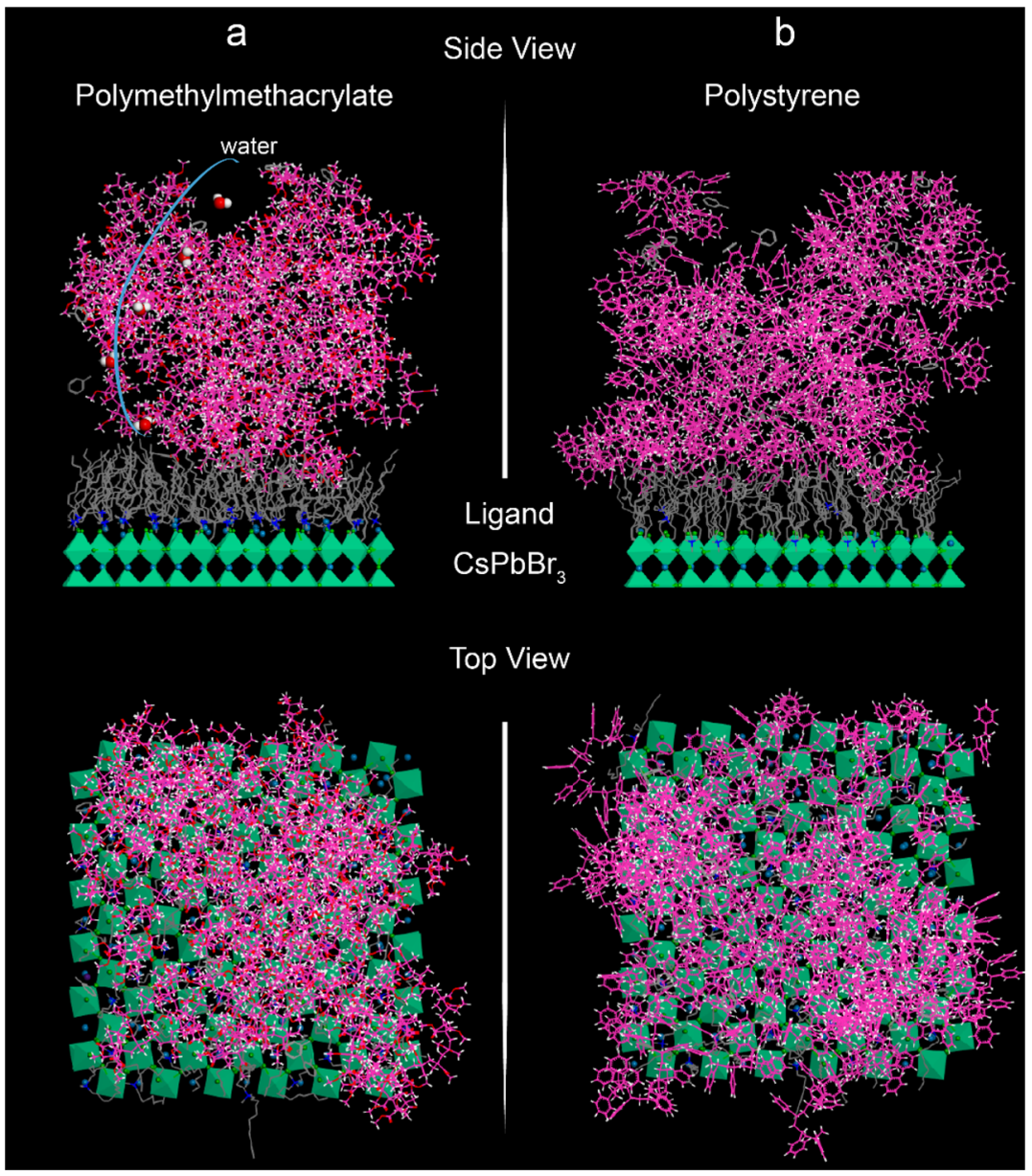

Figure 4. Molecular mechanics simulations for the NC ligands-polymer interaction in the case of PMMA (a) and PS (b). Side and top view images show that a denser surface coverage can be obtained with PS rather than PMMA.

coated on a glass substrate, such that NCs were directly exposed to air. To ensure that only one NC was probed, we performed photon correlation measurements in a HanburyBrown-Twiss setup. To study the photodegradation processes, consecutive PL spectra were continuously recorded with a $1 \mathrm{~s}$ integration time.

Figure 2 a reports the two-dimensional (2D) colored plot of consecutive PL spectra obtained on the reference sample of bare $\mathrm{CsPbX}_{3} \mathrm{NCs}$, that is, without the use of polymer encapsulation and hence with direct contact with air. As shown in Figure 2a, the PL spectrum experiences a dynamic blueshift with a concomitant quenching of the PL intensity, which is in good agreement with the observations by others. ${ }^{33,39}$ Antibunching behavior in the second-order correlation function (Figure $2 b$ ) attests that the observed behavior occurs at a single-particle level. The PL blueshift indicates that the effective size of the $\mathrm{NC}$ decreases due to decomposition. As reported for $\mathrm{CsPbI}_{3} \mathrm{NCs}$, etching of the $\mathrm{NC}$ surface occurs layer-by-layer (starting from the outside layer) at a constant rate, resulting in a steady reduction of the NC size. ${ }^{39}$ Perovskite NCs passivated with hydrophobic ligands are generally expected to be less sensitive to humidity compared to unprotected polycrystalline lead halide thin films. ${ }^{47}$ However, partial ligand coverage of the NC surface and the highly dynamic behavior of the ligands at the interface render perovskite NCs vulnerable when exposed to polar media. The study of the dynamic PL blueshift at the single particle level can be viewed as an accelerated stability test and may help reveal important information about the surface ligand and polymer coverage.

A standard practical strategy to reduce the detrimental effects of moisture and oxygen contaminants is the encapsulation of NCs in a protective matrix. At an ensemble level, recent attempts have already explored the use of hydrophobic polymers for enhancing the stability and the water resistance of perovskite compounds. ${ }^{47-52} \mathrm{We}$ have sampled different polymeric matrices, all dissolvable in the same apolar solvent as the NCs (i.e., toluene), a must-have prerequisite for the use as a matrix. These are PMMA, TOPAS, styrene-ethylene-butylene-styrene block copolymer (SEBS), and PS. Only PMMA and PS have thus far been known as matrices for single NC optical spectroscopy as they offer low autofluorescence. SEBS was recently employed at an ensemble level to increase the water resistance of $\mathrm{NC}$-polymer composites; in particular, films of $\mathrm{CsPbBr}_{3} \mathrm{NCs}$ have not lost their emissivity upon the direct immersion into the water for more than hundred days. ${ }^{47}$ TOPAS is used in diverse commercial applications, ranging from insulin delivery to food packaging films and mobile device components, owing to its very low water permeability and high optical transparency (see SI for a detailed comparison of different polymers).

To test the different polymers as protective matrices, a diluted solution of NCs was mixed with a solution of the polymer under test in toluene (see SI for more details). The 
final solution was then spin-coated on a glass coverslip. Ensemble PL and time-resolved PL for all NC-polymer composites are reported in the SI (Figure S1), along with a surface morphology characterization by atomic force microscopy (AFM), which reveals smooth and uniform films of about 70-90 $\mathrm{nm}$ in thickness (Figure S2).

Figure $3 \mathrm{a}$ reports the obtained consecutive $\mathrm{PL}$ spectra for a typical $\mathrm{CsPbBr}_{3} \mathrm{NC}$ in a PMMA matrix. It is evident that the PL experiences a similar temporal and irreversible blueshift, as already observed for the NCs directly exposed to air (Figure 2a). For each NC, we have conducted second-order correlation measurements to ensure that only single NCs were probed (Figure 3b). Results obtained on 20 different NCs (Figure 3c) show that all of them suffer from photodegradation with a typical degradation time (defined as the time at which the PL central peak has shifted by $10 \mathrm{~nm}$ ) on the order of few tens of seconds (see SI, Figure S3). Similar experiments using TOPAS (Figure $3 \mathrm{~d}-\mathrm{f}$ ) as a polymer matrix revealed minor improvements and the majority of NCs had still undergone a dynamic PL blueshift (Figure 3f) and a fast PL quenching. Contrary to the previously analyzed polymer matrices, NCs encapsulated in SEBS (Figure $3 \mathrm{~g}-\mathrm{i}$ ) showed enhanced optical stability and the majority of NCs had stable PL emission over the time window used in the experiments (Figure 3i). The best protective polymer matrix among those analyzed, turned out to be PS (Figure 3j-1), showing no detectable PL blueshift within 100 consecutive PL spectra (Figure 31). Second-order correlation data can be fitted by a series of exponential decay functions: we obtained an exciton lifetime in the order of $6 \mathrm{~ns}$ and a value for the $g 2(0)$ of about 0.1 (Figure $3 \mathrm{k}$ ), attesting the high purity single photon emission of perovskite NCs. Statistics on 30 different NCs (Figure 31) highlights the good ensemble uniformity. It is worth noting that stable NCs have an emission peak wavelength closer to the ensemble emission, while those experiencing strong dynamic PL blueshift are at shorter wavelengths already at time $=0$ (first frame in the PL series, Figure $3 \mathrm{c}, \mathrm{f}, \mathrm{i}, \mathrm{l})$. This attests that degradation due to the interaction with moisture and/or oxygen can be effective even under ambient light. A proper encapsulation, as observed by employing PS polymer, ensures stable emission with emission wavelengths closer to those observed in solution (Figure 1) or in ensemble NC-polymer composites (Figure S1).

The comparison of different polymeric matrices highlights that the polymer's water permeability is not the key parameter to avoid photodegradation. Indeed, all the studied polymers have low values of water permeability (see Table S1, SI), with TOPAS having 50 times lower water permeability coefficient as compared to PS (Table S1). However, good protection of the NC surface is guaranteed only if a good affinity exists between the polymer and NC ligands. In fact, TOPAS did not outperform PS as a protective matrix due to nonoptimal interaction with the nanoparticle surface (Figure S4).

The distinct behavior of $\mathrm{CsPbBr}_{3}$ NCs embedded in different polymers is puzzling and could only be rationalized after a closer look at the polymer chemical structures. We have performed molecular mechanics simulations of the polymerNC ligand interactions based on a force field approximation. The results obtained for PMMA and PS are reported in Figure 4. PMMA contains polar ester groups that are repelled by the highly hydrophobic ligands that surround the perovskite NCs. Hence, the polymer-NC interaction is rather poor and the encapsulation is therefore inefficient. Partial surface coverage is indeed obtained in our simulations, where we obtained for a fully relaxed ligand-polymer interface an enthalpy value of about $\Delta H \sim 27000 \mathrm{kcal} / \mathrm{mol}$ (see Figure 4a). Also, the polar groups of PMMA lead to higher retention of trace water content in the polymer under the equilibrium with ambient air (Table S1). To a certain extent, such moist PMMA could act as an etching agent favoring the desorption of $\mathrm{Pb}$ or $\mathrm{Br}$ atoms.

On the contrary, PS is a more hydrophobic polymer that contains aromatic rings and much lower residual equilibrium water content. PS molecules have higher affinity to the hydrophobic ligand shell of the NCs, which is mainly composed of oleic acid, oleylamine, and DDAB. Good surface coverage has been obtained in our simulations with a final enthalpy value of $\Delta H \sim 14000 \mathrm{kcal} / \mathrm{mol}$, for example, less positive (less destabilizing) than in the PMMA case, attesting the more favorable affinity between ligands and PS polymer (see Figure 4b).

In conclusion, our work demonstrates that specifics of the encapsulation of lead halide perovskite NCs into polymer matrix are of paramount importance for the observed optical behavior of these NCs at a single particle level. PS is the most effective matrix, whereas commonly used PMMA has a nonoptimal affinity to the hydrophobic NC ligands, higher residual water content under ambient conditions, and lower protection against environmental contaminants. Future work may benefit from the use of polymer matrices with tailored hydrophobicity via, for instance, the use of fluorinated groups, and with improved ligand-polymer interactions, expected with polymers grafted with long alkyl chains.

\section{ASSOCIATED CONTENT}

\section{Supporting Information}

The Supporting Information is available free of charge on the ACS Publications website at DOI: 10.1021/acs.nanolett.9b00689.

Detailed description of the NC colloidal synthesis, sample preparation, and experimental conditions; additional molecular mechanics simulations (PDF)

\section{AUTHOR INFORMATION}

\section{Corresponding Author}

*E-mail: mvkovalenko@ethz.ch.

ORCID

Stefan T. Ochsenbein: 0000-0002-5909-8650

Dmitry N. Dirin: 0000-0002-5187-4555

Maryna I. Bodnarchuk: 0000-0001-6597-3266

Maksym V. Kovalenko: 0000-0002-6396-8938

\section{Author Contributions}

The manuscript was written through the contributions of all authors. All authors have approved the final version of the manuscript.

\section{Notes}

The authors declare no competing financial interest.

\section{ACKNOWLEDGMENTS}

We acknowledge M. Grotevent for performing AFM measurements on NC-polymer composites. M.V.K. acknowledges financial support from the Swiss Federal Commission for Technology and Innovation (CTI-No. 18614.1 20 PFNMNM). 


\section{REFERENCES}

(1) Protesescu, L.; Yakunin, S.; Bodnarchuk, M. I.; Krieg, F.; Caputo, R.; Hendon, C. H.; Yang, R. X.; Walsh, A.; Kovalenko, M. V. Nano Lett. 2015, 15, 3692-3696.

(2) Akkerman, Q. A.; Gandini, M.; Di Stasio, F.; Rastogi, P.; Palazon, F.; Bertoni, G.; Ball, J. M.; Prato, M.; Petrozza, A.; Manna, L. Nat. Energy 2017, 2, 16194.

(3) Dutta, A.; Dutta, S. K.; Das Adhikari, S.; Pradhan, N. ACS Energy Lett. 2018, 3, 329-334.

(4) Imran, M.; Caligiuri, V.; Wang, M.; Goldoni, L.; Prato, M.; Krahne, R.; De Trizio, L.; Manna, L. J. Am. Chem. Soc. 2018, 140, $2656-2664$.

(5) Krieg, F.; Ochsenbein, S. T.; Yakunin, S.; ten Brinck, S.; Aellen, P.; Süess, A.; Clerc, B.; Guggisberg, D.; Nazarenko, O.; Shynkarenko, Y.; Kumar, S.; Shih, C.-J.; Infante, I.; Kovalenko, M. V. ACS Energy Lett. 2018, 3, 641-646.

(6) Levchuk, I.; Osvet, A.; Tang, X.; Brandl, M.; Perea, J. D.; Hoegl, F.; Matt, G. J.; Hock, R.; Batentschuk, M.; Brabec, C. J. Nano Lett. 2017, 17, 2765-2770.

(7) Song, J.; Li, J.; Xu, L.; Li, J.; Zhang, F.; Han, B.; Shan, Q.; Zeng,

H. Adv. Mater. 2018, 30, 1800764.

(8) Weidman, M. C.; Seitz, M.; Stranks, S. D.; Tisdale, W. A. ACS Nano 2016, 10, 7830-7839.

(9) Zhang, D.; Eaton, S. W.; Yu, Y.; Dou, L.; Yang, P. J. Am. Chem. Soc. 2015, 137, 9230-9233.

(10) Bodnarchuk, M. I.; Boehme, S. C.; ten Brinck, S.; Bernasconi, C.; Shynkarenko, Y.; Krieg, F.; Widmer, R.; Aeschlimann, B.; Günther, D.; Kovalenko, M. V.; Infante, I. ACS Energy Lett. 2019, 4, 63-74.

(11) Sutherland, B. R.; Sargent, E. H. Nat. Photonics 2016, 10, 295.

(12) Kovalenko, M. V.; Protesescu, L.; Bodnarchuk, M. I. Science 2017, 358, 745-750.

(13) Akkerman, Q. A.; Rainò, G.; Kovalenko, M. V.; Manna, L. Nat. Mater. 2018, 17, 394-405.

(14) Gangishetty, M. K.; Hou, S.; Quan, Q.; Congreve, D. N. Adv. Mater. 2018, 30, 1706226.

(15) Lignos, I.; Morad, V.; Shynkarenko, Y.; Bernasconi, C.; Maceiczyk, R. M.; Protesescu, L.; Bertolotti, F.; Kumar, S.; Ochsenbein, S. T.; Masciocchi, N.; Guagliardi, A.; Shih, C.-J.; Bodnarchuk, M. I.; deMello, A. J.; Kovalenko, M. V. ACS Nano 2018, $12,5504-5517$.

(16) Chiba, T.; Hayashi, Y.; Ebe, H.; Hoshi, K.; Sato, J.; Sato, S.; Pu, Y.-J.; Ohisa, S.; Kido, J. Nat. Photonics 2018, 12, 681-687.

(17) Chiba, T.; Hoshi, K.; Pu, Y.-J.; Takeda, Y.; Hayashi, Y.; Ohisa, S.; Kawata, S.; Kido, J. ACS Appl. Mater. Interfaces 2017, 9, 1805418060 .

(18) Li, J.; Xu, L.; Wang, T.; Song, J.; Chen, J.; Xue, J.; Dong, Y.; Cai, B.; Shan, Q.; Han, B.; Zeng, H. Adv. Mater. 2017, 29, 1603885.

(19) Veldhuis, S. A.; Boix, P. P.; Yantara, N.; Li, M.; Sum, T. C.; Mathews, N.; Mhaisalkar, S. G. Adv. Mater. 2016, 28, 6804-6834.

(20) Yakunin, S.; Protesescu, L.; Krieg, F.; Bodnarchuk, M. I.; Nedelcu, G.; Humer, M.; De Luca, G.; Fiebig, M.; Heiss, W.; Kovalenko, M. V. Nat. Commun. 2015, 6, 8056.

(21) Papagiorgis, P.; Manoli, A.; Protesescu, L.; Achilleos, C.; Violaris, M.; Nicolaides, K.; Trypiniotis, T.; Bodnarchuk, M. I.; Kovalenko, M. V.; Othonos, A.; Itskos, G. ACS Photonics 2018, 5, 907-917.

(22) Rainò, G.; Nedelcu, G.; Protesescu, L.; Bodnarchuk, M. I.; Kovalenko, M. V.; Mahrt, R. F.; Stöferle, T. ACS Nano 2016, 10, 2485-2490.

(23) Fu, M.; Tamarat, P.; Huang, H.; Even, J.; Rogach, A. L.; Lounis, B. Nano Lett. 2017, 17, 2895-2901.

(24) Isarov, M.; Tan, L. Z.; Bodnarchuk, M. I.; Kovalenko, M. V.; Rappe, A. M.; Lifshitz, E. Nano Lett. 2017, 17, 5020-5026.

(25) Becker, M. A.; Vaxenburg, R.; Nedelcu, G.; Sercel, P. C.; Shabaev, A.; Mehl, M. J.; Michopoulos, J. G.; Lambrakos, S. G.; Bernstein, N.; Lyons, J. L.; Stöferle, T.; Mahrt, R. F.; Kovalenko, M. V.; Norris, D. J.; Rainò, G.; Efros, A. L. Nature 2018, 553, 189-193.
(26) Ramade, J.; Andriambariarijaona, L. M.; Steinmetz, V.; Goubet, N.; Legrand, L.; Barisien, T.; Bernardot, F.; Testelin, C.; Lhuillier, E.; Bramati, A.; Chamarro, M. Nanoscale 2018, 10, 6393-6401.

(27) Ramade, J.; Andriambariarijaona, L. M.; Steinmetz, V.; Goubet, N.; Legrand, L.; Barisien, T.; Bernardot, F.; Testelin, C.; Lhuillier, E.; Bramati, A.; Chamarro, M. Appl. Phys. Lett. 2018, 112, 072104.

(28) Fu, M.; Tamarat, P.; Trebbia, J.-B.; Bodnarchuk, M. I.; Kovalenko, M. V.; Even, J.; Lounis, B. Nat. Commun. 2018, 9, 3318.

(29) Pfingsten, O.; Klein, J.; Protesescu, L.; Bodnarchuk, M. I.; Kovalenko, M. V.; Bacher, G. Nano Lett. 2018, 18, 4440-4446.

(30) Lin, X.; Dai, X.; Pu, C.; Deng, Y.; Niu, Y.; Tong, L.; Fang, W.; Jin, Y.; Peng, X. Nat. Commun. 2017, 8, 1132.

(31) Aharonovich, I.; Englund, D.; Toth, M. Nat. Photonics 2016, 10, 631.

(32) Hu, F.; Yin, C.; Zhang, H.; Sun, C.; Yu, W. W.; Zhang, C.; Wang, X.; Zhang, Y.; Xiao, M. Nano Lett. 2016, 16, 6425-6430.

(33) Park, Y.-S.; Guo, S.; Makarov, N. S.; Klimov, V. I. ACS Nano 2015, 9, 10386-10393.

(34) Li, B.; Huang, H.; Zhang, G.; Yang, C.; Guo, W.; Chen, R.; Qin, C.; Gao, Y.; Biju, V. P.; Rogach, A. L.; Xiao, L.; Jia, S. J. Phys. Chem. Lett. 2018, 9, 6934-6940.

(35) Tong, Y.; Fu, M.; Bladt, E.; Huang, H.; Richter, A. F.; Wang, K.; Müller-Buschbaum, P.; Bals, S.; Tamarat, P.; Lounis, B.; Feldmann, J.; Polavarapu, L. Angew. Chem., Int. Ed. 2018, 57, 16094-16098.

(36) Trinh, C. T.; Minh, D. N.; Ahn, K. J.; Kang, Y.; Lee, K.-G. ACS Photonics 2018, 5, 4937.

(37) Yarita, N.; Tahara, H.; Saruyama, M.; Kawawaki, T.; Sato, R.; Teranishi, T.; Kanemitsu, Y. J. Phys. Chem. Lett. 2017, 8, 6041-6047.

(38) Yarita, N.; Aharen, T.; Tahara, H.; Saruyama, M.; Kawawaki, T.; Sato, R.; Teranishi, T.; Kanemitsu, Y. Phys. Rev. Materials 2018, 2, 116003.

(39) Yuan, G.; Ritchie, C.; Ritter, M.; Murphy, S.; Gómez, D. E.; Mulvaney, P. J. J. Phys. Chem. C 2018, 122, 13407-13415.

(40) Gibson, N. A.; Koscher, B. A.; Alivisatos, A. P.; Leone, S. R. J. Phys. Chem. C 2018, 122, 12106-12113.

(41) Liu, L.; Deng, L.; Huang, S.; Zhang, P.; Linnros, J.; Zhong, H.; Sychugov, I. J. Phys. Chem. Lett. 2019, 10, 864-869.

(42) Chouhan, L.; Ghimire, S.; Biju, V. Angew. Chem. 2019, 131, 4929-4933.

(43) Huang, H.; Bodnarchuk, M. I.; Kershaw, S. V.; Kovalenko, M. V.; Rogach, A. L. ACS Energy Lett. 2017, 2, 2071-2083.

(44) Cho, H.; Kim, Y.-H.; Wolf, C.; Lee, H.-D.; Lee, T.-W. Adv Mater. 2018, 30, 1704587.

(45) Yang, D.; Li, X.; Zeng, H. Adv. Mater. Interfaces 2018, 5, 1701662.

(46) Luo, B.; Naghadeh, S. B.; Zhang, J. Z. ChemNanoMat 2017, 3, $456-465$

(47) Raja, S. N.; Bekenstein, Y.; Koc, M. A.; Fischer, S.; Zhang, D.; Lin, L.; Ritchie, R. O.; Yang, P.; Alivisatos, A. P. ACS Appl. Mater. Interfaces 2016, 8, 35523-35533.

(48) Hwang, I.; Jeong, I.; Lee, J.; Ko, M. J.; Yong, K. ACS Appl. Mater. Interfaces 2015, 7, 17330-17336.

(49) Huang, H.; Chen, B.; Wang, Z.; Hung, T. F.; Susha, A. S.; Zhong, H.; Rogach, A. L. Chem. Sci. 2016, 7, 5699-5703.

(50) Hai, J.; Li, H.; Zhao, Y.; Chen, F.; Peng, Y.; Wang, B. Chem. Commun. 2017, 53, 5400-5403.

(51) Zhang, H.; Wang, X.; Liao, Q.; Xu, Z.; Li, H.; Zheng, L.; Fu, H. Adv. Funct. Mater. 2017, 27, 1604382.

(52) Zhou, Q.; Bai, Z.; Lu, W.-g.; Wang, Y.; Zou, B.; Zhong, H. Adv. Mater. 2016, 28, 9163-9168. 